\title{
A Secure Mobile Authentication Alternative to Biometrics
}

\author{
Mozhgan Azimpourkivi \\ Florida International University \\ Miami, FL, USA
}

\author{
Umut Topkara \\ Bloomberg LP \\ New york, NY, USA
}

\author{
Bogdan Carbunar \\ Florida International University \\ Miami, FL, USA
}

\begin{abstract}
Biometrics are widely used for authentication in consumer devices and business settings as they provide sufficiently strong security, instant verification and convenience for users. However, biometrics are hard to keep secret, stolen biometrics pose lifelong security risks to users as they cannot be reset and re-issued, and transactions authenticated by biometrics across different systems are linkable and traceable back to the individual identity. In addition, their cost-benefit analysis does not include personal implications to users, who are least prepared for the imminent negative outcomes, and are not often given equally convenient alternative authentication options.

We introduce ai.lock, a secret image based authentication method for mobile devices which uses an imaging sensor to reliably extract authentication credentials similar to biometrics. Despite lacking the regularities of biometric image features, we show that ai.lock consistently extracts features across authentication attempts from general user captured images, to reconstruct credentials that can match and exceed the security of biometrics $(\mathrm{EER}=0.71 \%)$. ai.lock only stores a "hash" of the object's image. We measure the security of ai.lock against brute force attacks on more than 3.5 billion authentication instances built from more than 250,000 images of real objects, and 100,000 synthetically generated images using a generative adversarial network trained on object images. We show that the ai.lock Shannon entropy is superior to a fingerprint based authentication built into popular mobile devices.
\end{abstract}

\section{INTRODUCTION}

Existing solutions to the complex mobile authentication equation have significant problems. For instance, while biometric authentication provides sufficiently strong security, instant verification and convenience for users, biometrics are also hard to keep secret and pose lifelong security risks to users when stolen, as they cannot be reset and re-issued, More importantly, as surrendering biometrics may become de facto mandatory [34, 43], existing vulnerabilities $[5,21,51,71]$, coupled with the compromise of large scale biometrics databases [49], raise significant long term security concerns, especially as transactions authenticated by biometrics across different systems are linkable and traceable back to the individual identity. Further, token-based authentication solutions, e.g., SecurID [57], require an expensive infrastructure [1] (e.g. for issuing, managing, synchronizing the token).

A secret image based authentication approach, where users authenticate using arbitrary images they capture with the device camera, may address several of the above problems. For instance, the authentication is not tied to a visual of the user's body, but that of a personal accessory, object, or scene. As illustrated in Figure 1, a user sets her reference credential to be an image of a nearby object or scene. To authenticate, the user captures a candidate image; the authentication succeeds only if the candidate image contains the same object or scene as the reference image. This improves on (1) biometrics, by freeing users from personal harm, providing plausible deniability, allowing multiple keys, and making revocation and change of secret simple and (2) token-based authentication, by eliminating the need for an expensive infrastructure. Visual tokenbased solutions (e.g., based on barcodes or QR codes) [24, 41] can be seen as special cases of secret image based authentication.

However, this approach raises new challenges. First, an adversary who captures or compromises the device that stores the user's reference credentials (e.g. mobile device, remote server) and has access to its storage, should not be able to learn information about the reference credentials or their features. Second, while biometric features such as ridge flow of fingerprints or eye socket contours of faces, can be captured with engineered features and are invariant for a given user, images of objects and general scenes lack a well defined set of features that can be accurately used for authentication purposes. Improper features will generate (i) high false accept rates (FAR), e.g., due to non-similar images with similar feature values, and (ii) high false reject rates (FRR) that occur due to angle, distance and illumination changes between the capture circumstances of reference and candidate images.

In a first contribution, we introduce ai.lock, a practical, secure and efficient image based authentication system that converts general mobile device captured images into biometric-like structures, to be used in conjunction with secure sketch constructs and provide secure authentication and storage of credentials [ $\$ 5]$.

To extract invariant features for image based authentication, ai.lock leverages (1) the ability of Deep Neural Networks (DNNs) to learn representations of the input space (i.e., embedding vectors of images) that reflect the salient underlying explanatory factors of the data, (2) Principal Component Analysis (PCA) [20] to identify more distinguishing components of the embedding vectors and (3) Locality Sensitive Hashing (LSH) to map the resulting components to binary space, while preserving similarity properties in the input space. We call the resulting binary values imageprints. ai.lock builds on a secure sketch variant [17] to securely store reference imageprints and match them to candidate imageprints.

In a second contribution, we propose the LSH-inspired notion of locality sensitive image mapping functions ( $\delta$-LSIM), that convert images to binary strings that preserve the "similarity" relationships of the input space, for a desired similarity definition [ $§ 3]$. A $\delta$-LSIM function can be used to efficiently match images based on their extracted binary imageprints.

Further, we develop brute force image based attacks that aim to defeat ai.lock. First, we perform real image attacks, that use manually collected and publicly available image datasets. To evaluate ai.lock on large scale attack images, we develop synthetic image attacks that use images produced by generative models [54]. To evaluate the resilience of stored credentials, we introduce synthetic credential attacks, that use authentication credentials generated with 


\begin{tabular}{lcccc}
\hline Solution & $\begin{array}{c}\text { FAR } \\
\mathbf{( \% )}\end{array}$ & $\begin{array}{c}\text { EER } \\
\mathbf{( \% )}\end{array}$ & $\begin{array}{c}\text { Shannon Entropy } \\
\text { (bits) }\end{array}$ & $\begin{array}{c}\text { Dataset } \\
\text { size }\end{array}$ \\
\hline ai.lock (MLMS) & 0.0004 & - & 18.02 & $2 \times 10^{9}$ \\
ai.lock (MLSS) & 0.0015 & 0.17 & 16.02 & $6 \times 10^{6}$ \\
\hline iPhone TouchID [4] & 0.0020 & - & 15.61 & - \\
\hline Deepface [65] (face) & - & 8.6 & - & $>0.5 \times 10^{9}$ \\
SoundProof [33] (sound) & 0.1 & 0.2 & 9.97 & $>2 \times 10^{6}$ \\
[62] (eye movement) & 0.06 & 6.2 & 10.70 & 1,602 \\
\hline RSA SecurlD [57] & - & - & 19.93 & - \\
\hline Text-based password [10] & - & - & $10-20$ & $7 \times 10^{7}$ \\
\hline
\end{tabular}

Table 1: ai.lock variants vs. commercial and academic biometric, token-based authentication solutions, and text passwords. ai.lock MLSS variant has no false rejects, as it is evaluated under attack samples only. Under large scale datasets of powerful attacks, ai.lock achieves better entropy than stateof-the-art biometric solutions.

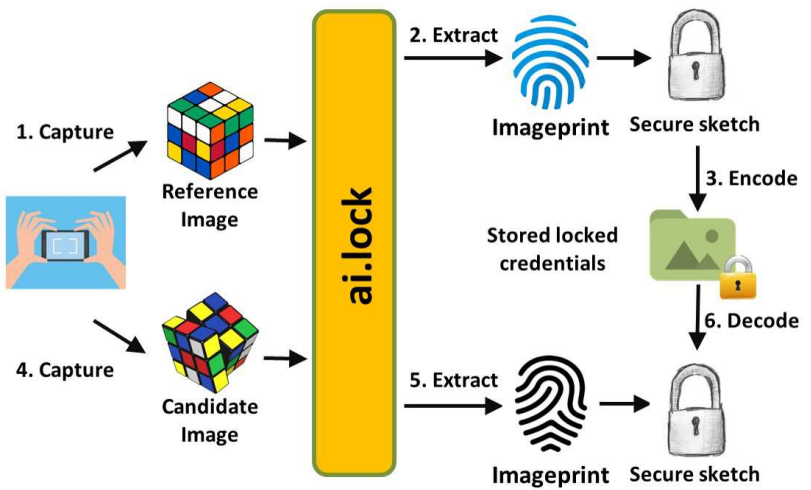

Figure 1: ai.lock model and scenario. The user captures the image of an object or scene with the device camera. ai.lock converts the image to a binary imageprint, and uses it as a biometric, in conjunction with a secure sketch solution, to securely store authentication information on the device or on a remote server. The user can authenticate only if she is able to capture another image of the same object or scene.

the same distribution of the credentials extracted from manually collected images [\$2.1]. We have captured, collected and generated datasets of 250,332 images, and generated 1 million synthetic credentials [ $\S 6.1]$. We have used these datasets to generate attack datasets containing more than 3.5 billion $(3,567,458,830)$ authentication instances [\$6.2].

We have implemented an ai.lock in Android using Tensorflow [3] and show that it is resilient to attacks. Its FAR on 140 million synthetic image attack samples is $0.2 \times 10^{-6} \%$. ai.lock was unbreakable when tested with 1.4 billion synthetic credential attack samples. The estimated Shannon entropy [60] of ai.lock on 2 billion image pairs is 18.02 bits, comparing favorably with state-of-the-art biometric solutions (see Table 1). Further, we show that ai.lock is a $\delta$ LSIM function, over images that we collected [\$ 7.4]. ai.lock is fast, imposing an overhead of under 1s on a Nexus 6P device. We have released the code and data on https://github.com/casprlab/ai.lock.

\section{MODEL AND APPLICATIONS}

We consider a user that has a camera equipped device, e.g., smartphone or tablet, a resource constrained device such as a smart

watch/glasses, or a complex cyber-physical system such as a car. The user needs to authenticate to the device or an application backend, or authenticate through the device to a remote service. For this, we assume that the user can select and easily access a physical object or scene. To set her password, the user captures the image of an object/scene with the device camera, see Figure 1 for an illustration. ai.lock extracts a set of features from the user's captured reference image, then stores this information (imageprint) securely either on the device or on a remote server. We note that, in the former case, the device can associate the reference image with the user's authentication credentials (e.g. OAuth [14]) for multiple remote services. To authenticate, the user needs to capture another image. The user is able to authenticate only if the candidate image is of the same object or scene as the reference image. Similar to e.g., text passwords, the user can choose to reuse objects across multiple services, or use a unique object per service. Using a unique object per service will affect memorability. However, due to the image superiority effect [45], objects may be easier to remember than text passwords. In the following, we describe a few applications of this model.

Alternative to biometric authentication. Instead of authenticating with her sensitive and non-replaceable biometrics (face, fingerprint), the user uses a unique nearby scene or object that she carries, e.g., a trinket, Rubik's cube with a unique pattern, printed random art, etc. ai.lock moves the source of information from the user to an externality, as it does not require a visual of the user's body, but that of a personal accessory, object, or scene that the user can recreate at authentication time. ai.lock improves on biometrics by freeing users from personal harm, providing plausible deniability, allowing multiple keys, and making revocation and change of secret simple.

Location based authentication. The user chooses as password an image of a unique scene at a frequented location (office, home, coffee shop), e.g., section of book shelf, painting, desk clutter. This approach can be generalized to enable location based access control, e.g., to provide restricted access to files and networks in less secure locations.

Cyber-physical system authentication. Our model supports authentication to cyber-physical systems, including car and door locks, thermostat and alarm systems, where key and PIN entry hardware $[58,59]$ is replaced with a camera. To authenticate, the user needs to present her unique but replaceable authentication object to the camera.

\subsection{Adversary Model}

We assume an active adversary who can physically capture or compromise the device that stores the user credentials. Such an adversary can not only access the stored credentials, but also any keying material stored on the device, then use it to recover encrypted data and use it to authenticate through the proper channels. However, we assume that the adversary does not have control over the authentication device while the user authenticates (e.g., by installing malware). We also assume an adversary with incomplete surveillance [19], i.e., who can physically observe the victim during authentication but cannot capture the details of the secret object. 
Furthermore, we assume that the adversary has "blackbox access" to the authentication solution, thus can efficiently feed it images of his choice and capture the corresponding imageprint. The adversary can use this output to learn information from the stored credentials. More specifically, we consider the following attacks:

- Real image attack. The adversary collects large datasets of images, e.g., manually using a mobile camera, and online. Then, in a brute force approach, he matches each image as an authentication instance against the stored reference credentials until success.

- Synthetic image attack. The adversary uses the previously collected images to train a generative model, e.g. [23], that captures essential traits of the images, then uses the trained model to generate a large dataset of synthetic images. Finally, the adversary matches each such image against the reference credentials.

- Synthetic credential attack. Instead of images, the adversary queries the authentication system with binary imageprints. For this, the adversary extracts the imageprints generated by the authentication solution on real images of his choice. He then generates a large dataset of synthetic credentials that follow the same distribution as the extracted credentials. Finally, he matches each synthetic credential exhaustively against the reference credentials.

- Object/scene guessing attack. While we do not consider shoulder surfing attacks which also apply to face based authentication $[35,71]$, we assume an adversary that is able to guess the victim's secret object/scene type. The adversary then collects a dataset of images containing the same object or scene type, then uses them to brute force ai.lock (see Appendix B).

Finally, we assume the use of standard secure communication channels for the remote authentication scenario where the user credentials are stored on a server.

\section{PROBLEM DEFINITION}

Let II denote the space of images that can be captured by a user with a camera. Let $\operatorname{sim}: \mathbb{I} \times \mathbb{I} \rightarrow\{0,1\}$ be a function that returns true when its input images have been taken with the same camera and are of the same object or scene, and false otherwise.

Informally, the image based authentication problem seeks to identify a store function $S: \mathbb{I} \rightarrow\{0,1\}^{k}$, and an authentication function Auth $:\{0,1\}^{k} \times\{0,1\}^{*} \rightarrow\{0,1\}$ (for a parameter $k$ ) that satisfy the following properties. First, it is hard for any adversary with access to only $S(R)$, for a reference image $R \in \mathbb{I}$, to learn information about $R$. That is, $S$ imposes a small entropy reduction on its input image. Second, for any candidate string $C \in\{0,1\}^{*}, \operatorname{Auth}(S(R), C)=1$ only if $C \in \mathbb{I}$ and $\operatorname{sim}(R, C)=1$. Thus, a candidate input to the Auth function succeeds only if it is a camera captured image of the same object or scene as the reference image.

We observe that the secure sketch of [17] solves this problem for biometrics: given a biometric input, the secure sketch outputs a value that reveals little about the input, but allows its reconstruction from another biometric input that is "similar". Therefore, the image based authentication problem can be reduced to the problem of transforming camera captured images of arbitrary objects and scenes into biometric-like structures.

Hence, we introduce the LSH-related notion of locality sensitive image mapping functions. Specifically, let $d:\{0,1\}^{\lambda} \times\{0,1\}^{\lambda} \rightarrow \mathbb{R}$ be a distance function (e.g., Hamming), where $\lambda$ is a system parameter. Then, for a given $\delta \in[0,1]$, a $\delta$-Locality Sensitive Image Mapping (LSIM) function $h$ satisfies the following properties:

Definition 3.1. $h: \mathbb{I} \rightarrow\{0,1\}^{\lambda}$ is a $\delta$-LSIM function if there exist probabilities $P_{1}$ and $P_{2}, P_{1}>P_{2}$, s.t.:

(1) For any two images $I_{1}, I_{2} \in \mathbb{I}$, if $\operatorname{sim}\left(I_{1}, I_{2}\right)=$ true, then $\frac{d\left(h\left(I_{1}\right), h\left(I_{2}\right)\right)}{\lambda}<\delta$ with probability $P_{1}$.

(2) For any two images $I_{1}, I_{2} \in \mathbb{I}$, if $\operatorname{sim}\left(I_{1}, I_{2}\right)=$ false, then $\frac{d\left(h\left(I_{1}\right), h\left(I_{2}\right)\right)}{\lambda}>\delta$ with probability $P_{2}$.

\section{BACKGROUND \& RELATED WORK}

To build ai.lock we leverage deep learning based feature extraction, locality sensitive hashing and secure sketch constructs. In the following, we briefly describe these concepts.

\subsection{Biometric Protection}

Our work is related to the problem of protecting biometric templates. We summarize biometric protection solutions, that can be classified into fuzzy biometric protection and feature transformation approaches [29].

Fuzzy biometric template protection. This approach leverages error correcting codes to verify biometric data. Techniques include secure sketch and fuzzy extractor [17], fuzzy vault [31] and fuzzy commitment [32], and have been applied to different biometric data, e.g. palm and hand [37].

In this paper, we extend the secure sketch under the Hamming distance solution from [17]: reconstruct the biometric credential, then compare its hash against a stored value. We briefly describe here the password set and authentication procedures that we use based on ai.lock generated imageprints (see $\S 5$ ). Let ECC be a binary error correcting code, with the corresponding decoding function $D$, and let $H$ be a cryptographic hash function.

- Image password set. Let $R$ be the reference image captured by the user and let $\pi_{R}=\pi(R)$ be its ai.lock computed imageprint. Generate a random vector $x$, then compute and store the authentication credentials, $S S(R, x)=\left\langle S S_{1}, S S_{2}\right\rangle$, where $S S_{1}=\pi_{R} \oplus E C C(x)$ and $S S_{2}=H(x)$.

- Images based authentication. Let $C$ be the user captured candidate image, and let $\pi_{C}=\pi(C)$ be its ai.lock computed imageprint $(\S 5)$. Retrieve the stored $S S$ value and compute $x^{\prime}=$ $D\left(\pi_{C} \oplus S S_{1}\right)$. The authentication succeeds if $H\left(x^{\prime}\right)=S S_{2}$.

Transformation based biometric template protection. A transformation is applied both to the biometric template and the biometric candidate, and the matching process is performed on the transformed data. In an invertible transformation (a.k.a., salting [29]), a key, e.g., a password, is used as a parameter to define the transformation function [68]. The security of this approach depends on the ability to protect the key. In contrast, in non-invertible schemes $[40,56]$ a one-way transformation functions is used to protect the biometric template, making the inversion of a transformed template computationally hard even when the key is revealed.

Hybrid approaches. Hybrid transformation and fuzzy protection approaches have also been proposed. Nandakumar et al. [44] introduced an approach to make the fingerprint fuzzy value stronger using a password as salt. Song et al. [47] used discrete hashing 
to transform the fingerprint biometric, which is then encoded and verified using error correcting codes.

\subsection{Deep Neural Networks (DNNs)}

Empirical results have shown the effectiveness of representations learned by DNNs for image classification [18, 52, 72], and for the verification of different biometric information [13, 42, 48]. However, ai.lock differs in its need to ensure that two object images contain the exact same object, for the purpose of authentication. ai.lock exploits the ability of DNNs to learn features of the input image space that capture the important underlying explanatory factors. We conjecture that these features will have small variations among images of the same object or scene, captured in different circumstances.

Pretrained Inception.v3. Training a DNN with millions of parameters is computationally expensive and requires a large training dataset of labeled data, rarely available in practice. Instead, we employed a transfer learning [61] approach: obtain a trained DNN and use it for a similar task. For image feature extraction, we use Inception.v3 [64] network pretrained on ImageNet dataset [16], of 1.2 million images of 1,000 different object categories, for image classification.

\subsection{Locality Sensitive Hashing}

Locality Sensitive Hashing (LSH) seeks to reduce the dimensionality of data, while probabilistically preserving the distance properties of the input space. It was initially used to solve the near neighbor search problem in high dimensional spaces [28]. While seemingly the ideal candidate to provide the ai.lock functionality, LSH does not work well on images: images of the same scene or object, captured in different conditions, e.g., angle, distance, illumination, will have dramatically different pixel values, leading to a high distance between the images and thus also between their LSH values.

We use however Charikar's [12] LSH as a building block in ai.lock. Charikar's [12] LSH defines a family of hash functions in the space $\mathcal{R}^{d}$. Specifically, the LSH function $h_{r}$ is based on a randomly chosen $d$-dimensional Gaussian vector with independent components $r \in \mathcal{R}^{d}$, where $h_{r}(u)=1$ if $r \cdot u \geq 0$ and $h_{r}(u)=0$ if $r \cdot u<0$, where $\cdot$ denotes the inner product. This function provides the property that $\operatorname{Pr}\left[h_{r}(u)=h_{r}(v)\right]=1-\frac{\theta(u, v)}{\pi}$, for any vectors $u$ and $v$, where $\theta(u, v)$ denotes the angle between the input vectors.

\subsection{Privacy Preserving Image Matching}

Traditional approaches to object matching and object recognition, e.g., SIFT [39] and SURF [8], rely on extracting identifying features or (robust/invariant) keypoints and their descriptors at specific locations on the image. Several solutions have been proposed for the secure image matching problem that could be applied to the image based authentication task. SecSIFT [53] employed orderpreserving encryption and distributes the SIFT computation among different servers. Hsu et al. [27] proposed a privacy preserving SIFT based on homomorphic encryption, while Bai et al. [7] performed SURF feature extraction in encrypted domain using Paillier's homomorphic cryptosystem. Wang et al. [70] improve the SURF algorithm in encrypted domain by designing protocols for

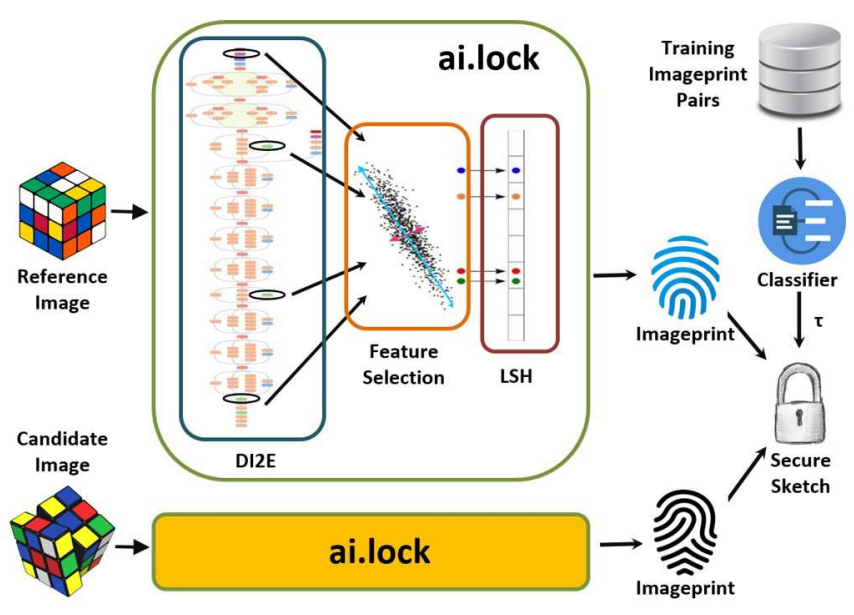

Figure 2: ai.lock architecture. ai.lock processes the input image through a deep neural network (i.e., Inception.v3), selects relevant features, then uses locality sensitive hashing to map them to a binary imageprint. ai.lock uses a classifier to identify the ideal error tolerance threshold $(\tau)$, used by the secure sketch block to lock and match imageprints.

secure multiplication and comparison, that employ a "somewhat" homomorphic encryption. These approaches are not practical on mobile devices, due to the high cost of homomorphic encryption and the large number of keypoints (up to thousands per image).

\subsection{Token-Based Authentication}

In previous work [6] we have evaluated the usability of Pixie, a trinket based authentication solution that employs slightly outdated image processing techniques to extract features (i.e., "keypoints") and match user captured images. Pixie has an important drawback when deployed on mobile devices: the image keypoints that it extracts need to be stored and matched in cleartext on vulnerable devices. In contrast, ai.lock uses state of the art, deep neural network based image feature extraction along with LSH to extract binary imageprints that are robust to changes in image capture conditions. The imageprints can be securely stored and matched using secure sketches. This makes ai.lock resilient to device capture attacks. Furthermore, on larger and more complex attack datasets, the use of DNNs enabled ai.lock to achieve false accept rates that are at least 2 orders of magnitude smaller than those of Pixie ( $\leq 0.0015 \%$ vs. $0.2-0.8 \%$ ), for similar FRRs (4\%).

ai.lock's secret physical object is similar to token-based authentication, either hardware or software. For instance, SecurID [57] generates pseudo-random, 6 digit authentication codes. ai.lock's Shannon entropy is slightly lower than SecurID's 19.93 bits (see Table 1 for comparison). Several authentication solutions use visual tokens (e.g., barcodes or QR codes). For instance, McCune, et al. [41] proposed Seeing-is-Believing, a schema that relies on a visual authentication channel that is realized through scanning a barcode. Hayashi et al. [24] introduced WebTicket, a web account management system that asks the user to print or store a 2D barcode on a secondary device and present it to the authentication device's webcam in order to authenticate to a remote service. Tokenbased authentication requires an expensive infrastructure [1] (e.g. 


\begin{tabular}{c|l}
\hline Symbol & Description \\
\hline$\lambda$ & Length of the imageprint for a single image segment \\
$\tau$ & Error tolerance threshold \\
$c$ & Correctable number of bits \\
$s$ & Number of image segments in multi segment schema \\
$t$ & Segment-based secret sharing threshold \\
\hline
\end{tabular}

Table 2: ai.lock notations.

for issuing, managing, synchronizing the token). ai.lock provides a "hash-like" construct for arbitrary object images, making objects usable as passwords, with the existing infrastructure.

Other approaches exist that seek to transform biometrics into tokens that the user needs to carry, with important implications on biometric privacy and revocation capabilities. For instance, TAPS [2] is a glove sticker with a unique fingerprint intended for TouchID.

\section{THE AI.LOCK SOLUTION}

We introduce ai.lock, the first locality sensitive image mapping function, and a practical image based authentication system. In the following, we describe the basic solution, then introduce two performance enhancing extensions.

\section{1 ai.lock: The Basic (SLSS) Solution}

ai.lock consists of 3 main modules (see Figure 2): (1) deep image-toembedding (DI2E) conversion module (2) feature selection module, and (3) LSH based binary mapping module. We now describe each module and its interface with the secure sketch module (see $§ 4.1$ ). Table 2 summarizes the important ai.lock parameter notations.

Deep image to embedding (DI2E) module. Let $I$ be the fixed size input image. Let $E m b: \mathcal{I} \rightarrow \mathcal{R}^{e}$ be a function that converts images into feature vectors of size $e$. We call $\operatorname{Emb}(I)$ the embedding vector, an abstract representation of $I$. To extract $E m b(I)$, ai.lock uses the activations of a certain layer of Inception.v3 DNN [64] when $I$ is the input to the network. Let $e$ denote the size of the output of the layer of the DNN used by ai.lock. Thus, $\operatorname{Emb}(I) \in \mathcal{R}^{e}$. Feature selection module. We have observed that not all the components in the embedding feature vectors are relevant to our task (see $\S 7.1$ ). Therefore, we reduce the dimensionality of the feature vectors to improve the performance and decrease the processing burden of ai.lock. Let $P: \mathcal{R}^{e} \rightarrow \mathcal{R}^{p}$, where $p<e$ be a function that reduces the features of an embedding to the ones that are most important. ai.lock uses PCA with component range selection as the $P$ function, and applies it to $E m b(I)$ to find a set of components that can reflect the distinguishing features of images. Thus, the vector produced by feature selection module is $P(\operatorname{Emb}(I)) \in \mathcal{R}^{p}$.

LSH based binary mapping module. In a third step, ai.lock seeks to map $P(E m b(I))$ to a binary space of size $\lambda$ that preserves the similarity properties of the input space. To address this problem, we use the LSH scheme proposed by Charikar [12]. Let $L$ : $\mathcal{R}^{p} \rightarrow\{0,1\}^{\lambda}$ be such a mapping function. ai.lock uses as $L$, a random binary projection LSH as follows. Let $M$ be a matrix of size $p \times \lambda$, i.e. $\lambda$ randomly chosen $p$-dimensional Gaussian vectors with independent components. Calculate $b$ as the dot product of $P(\operatorname{Emb}(I))$ and $M$. For each coordinate of $b$, output either 0 or 1 , based on the sign of the value of the coordinate. We call this binary representation of the input image $I$, i.e. $\pi(I)=L(P(E m b(I)))$,

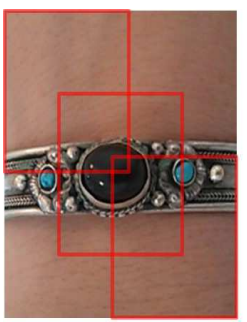

(a)
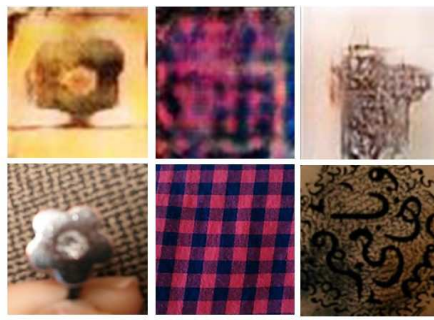

(b)
Figure 3: (a) 3 overlapping segments of an image. (b) Top: sample images generated by DCGAN, Bottom: visually similar images in Nexus Dataset to images generated by DCGAN.

its imageprint. We denote the length of a single imageprint by $\lambda$. Note that, the hash value for the Charikar's method is a single bit $(\lambda=1)$. Therefore, $L$ can be viewed as a function that returns a concatenation of $\lambda$ such random projection bits.

In $\S 7.4$ we provide empirical evidence that the function $h=$ $L \circ P \circ E m b$ is a $(\tau)$-LSIM transform (see $\S 7.1$ ), for specific $\tau$ values. Secure sketch. ai.lock extends the secure sketch under the Hamming distance of [17] to securely store the binary imageprint credentials and perform image password set and image based authentication as described in $\S 4.1$.

In the following, we introduce two ai.lock extensions, intended to increase the entropy provided by ai.lock's output. First, we modify ai.lock to use the embedding vectors obtained from multiple layers of Inception.v3 network. Second, we extend ai.lock to split the input image into multiple overlapping segments and concatenate their resulting binary representations.

\section{2 ai.lock with Multiple DNN Layers}

Representations learned by a DNN are distributed in different layers of these networks. The lower (initial) layers of convolutional neural networks learn low level filters (e.g. lines, edges), while deeper layers learn more abstract representations [73]. The use of a single DNN layer prevents the basic ai.lock solution from taking advantage of both filters.

To address this issue, we propose an ai.lock extension that collects the embedding vectors from multiple $(l)$ layers of Inception.v3 network. In addition, we modify the basic ai.lock feature extractor module as follows. The Principal Components (PCs) of activations for each layer are computed separately and are mapped to a separate binary string of length $\lambda$. Then, the binary strings constructed from different layers are concatenated to create a single imageprint for the input image. Thus, the length of the imageprint increases linearly with the number of layers used in this schema.

\section{3 ai.lock with Multiple Image Segments}

We divide the original image into $s$ overlapping segments (see Figure 3(a)). We then run the basic ai.lock over each segment separately to produce $s$ different imageprints of length $\lambda$. However, we identify the PCs for the embedding vectors of each segment based on the whole size images. The intuition for this choice is that random image segments are not good samples of real objects and may confuse the PCA. We then generate the imageprint of the original, 
whole size image, as the concatenation of the imageprints of its segments.

Secure sketch sharing. We extend the secure sketch solution with a $(t, s)$-secret sharing scheme. Specifically, let $x_{1}, \ldots, x_{s}$ be $(t, s)$-shares of the random $x$, i.e., given any $t$ shares, one can reconstruct $x$. Given a reference image $R$, let $R^{(1)}, . . R^{(s)}$ be its segments, and let $\pi_{R}^{(i)}=\pi\left(R^{(i)}\right), i=1$..s be their imageprints. Then, we store $S S(R, x)=\left\langle S S_{1}^{(1)}, \ldots, S S_{1}^{(s)}, S S_{2}\right\rangle$, where $S S_{1}^{(i)}=\pi_{R}^{(i)} \oplus E C C\left(x_{i}\right)$ and $S S_{2}=H(x)$. To authenticate, the user needs to provide a candidate image $C$, whose segments $C^{(i)}, i=1 . . s$ produce imageprints $\pi_{C}^{(i)}=\pi\left(C^{(i)}\right)$ that are able to recover at least $t$ of $x$ 's shares $x_{i}$.

\section{IMPLEMENTATION \& DATA}

We build ai.lock on top of the Tensorflow implementation for Inception.v3 network [67]. For the error correcting code of secure sketches, we use a $\mathrm{BCH}[11,26]$ open source library [30], for syndrome computation and syndrome decoding with correction capacity of up to $c$ bits. The value for $c$ is calculated empirically using the training dataset (see $§ 7.1$ )

Basic (SLSS) ai.lock. In the basic ai.lock solution, we use the output of the last hidden layer of Inception.v3 network, before the softmax classifier, consisting of 2, 048 float values. Our intuition is that this layer provides a compact representation (set of features) for the input image objects, that is efficiently separable by the softmax classifier.

Multi layer ai.lock. For the multi DNN layer ai.lock variants, we have used 2 layers $(l=2)$. The first layer is the "Mixed_8/Pool_0" layer and the second layer is the last hidden layer in Inception.v3. The embedding vector for the "Mixed_8/Pool_0" consists of 49, 152 float values. As described in $\S 5.3$, the embedding vectors of each layer are separately processed by the feature selection and LSH modules; the resulting binary strings are concatenated to form the imageprint of size $2 \lambda$.

Multi segment ai.lock. For the multi segment ai.lock variant, we split the image into multiple segments that we process independently. Particularly, we consider 5 overlapping segments, cropped from the top-left, bottom-left, top-right, bottom-right and the center of the image. We generate segments whose width and height is equal to the width and height of the initial image divided by 2 , plus 50 pixels to ensure overlap. The extra 50 pixels are added to the interior sides for the side segments. For the middle segment, 25 pixels are added to each of its sides. Each segment is then independently processed with the basic ai.lock (i.e., last hidden layer of Inception.v3, PCA, LSH).

Multi layer multi segment ai.lock. This is a hybrid of the above variants: split the image into 5 overlapping parts, then process each part through Inception.v3 network, and extract the activation vectors for each of the two layers of Inception.v3 (the last hidden layer and Mixed_8/Pool_0 layer). The output of each layer for each segment is separately processed as in the basic ai.lock. Thus, the resulting imageprint of the image has $10 \lambda$ bits.

\subsection{Primary Data Sets}

6.1.1 Real Images.

Nexus dataset. We have used a Nexus 4 device to capture 1,400 photos of 350 objects, belonging to 33 object categories. Example of object categories in this dataset includes watches, shoes, jewelry, shirt patterns, and credit cards. We have captured 4 images of each object, that differ in background and lighting conditions.

ALOI dataset. We have used the "illumination direction" subset of the Amsterdam Library of Object Images (ALOI) [22] dataset. This dataset includes 24 different images of 1000 unique objects $(24,000$ in total) that are taken under different illumination angles.

Google dataset. We have used Google's image search to retrieve at least 200 images from each of the 33 object categories of the Nexus image dataset, for a total of 7,853 images. This dataset forms the basis of a "targeted" attack.

YFCC100M toy dataset. We have extracted a subset of the Yahoo Flickr Creative Commons 100M (YFCC100M) [69] image dataset (100 million Flickr images), of 126,600 Flickr images tagged with the "toy" keyword, and not with "human" or "animal" keywords.

\subsubsection{Synthetic Data.}

Synthetic image dataset. Manually capturing the Nexus dataset was a difficult and time consuming process. In order to efficiently generate a large dataset of similar images, we have leveraged the ability of generative models to discover an abstract representation that captures the essence of the training samples. Generative models, including Generative Adversarial Networks (GAN) [23], are trained to generate samples that are similar to the data they have been trained on. Such models have been shown to be suitable for representation learning tasks, e.g., [54].

We have used a DCGAN network [54] to generate a large set of synthetic images that are similar to the images in the Nexus dataset. Specifically, we have trained a DCGAN network [54] using the images of the Nexus dataset for 100 training epochs. Image augmentation, e.g., rotation, enhancement, and zoom, is performed to artificially increase the number of Nexus image dataset samples to include 20 variants per image. We then used the trained network to generate synthetic images: generate a random vector $(z)$ drawn from the uniform distribution, then feed $z$ to DCGAN's generator network to construct an image. We repeated this process to generate 200,000 images, that form our synthetic image dataset. Figure 3(b) shows sample images generated by this network, alongside similar images from the Nexus dataset.

Synthetic credential dataset. We have generated the binary imageprints for the images in Nexus dataset based on the best parameters of ai.lock (see $\S 7.1$ ). For each considered $\lambda$ value, we consider the value at each position of the binary imageprint as an independent Bernoulli random variable. We then calculate the probability of observing a 1 in each position based on the imageprints of the Nexus dataset. We use these probabilities to draw 100,000 random samples (of length $\lambda$ ) from the corresponding Bernoulli distribution for each position. The resulting random binary imageprints form our synthetic credential dataset. We have experimented with 10 values of $\lambda$ ranging from 50 to 500 , thus, this dataset contains 1 million synthetic imageprints.

\subsection{Evaluation Datasets}

We use the above image datasets to generate authentication samples that consist of one candidate image and one reference image. 
ai.lock attack dataset. We use roughly $85 \%$ of the images from the Nexus, ALOI and Google datasets as a training set to train and estimate the performance of ai.lock. We use the remaining $15 \%$ of images in each dataset (i.e., 220 Nexus, 3,600 ALOI and 1,178 Google images) as a holdout set. We use the holdout dataset to assess the generalization error of the final model, and as a real image attack dataset (see $\S 7.3$ ).

We generate the samples in holdout dataset using each subset of Nexus, ALOI and Google separately as follows. Each image of the Nexus holdout dataset is chosen as a reference image once, then coupled once with all the other images in the Nexus, ALOI and Google sets, used as candidate images. Therefore, there are $\frac{220 \times 219}{2}=24,090$ combinations of samples for the images in the Nexus set. For each 55 unique objects in this set, there are $6\left(\left(\begin{array}{l}4 \\ 2\end{array}\right)\right)$ possible valid samples that compare one image of this object to another image of the same object. Thus, there are $55 \times 6=330$ valid samples in the Nexus set. We then generate $220 \times 3,600=792 \mathrm{~K}$ and $220 \times 1,178=259,160$ invalid samples from comparing Nexus images to images in ALOI and Google sets respectively. Therefore, the ai.lock holdout set contains a total of 1, 075, 250 samples.

In addition, the training set is further divided into 5 folds, for cross validation. Each training fold contains 236, 4, 080 and 1, 335 images of Nexus, ALOI and Google datasets respectively. Therefore, there are $\frac{236 \times 235}{2}=27,730$ samples for the fold's 59 unique Nexus set objects, of which $59 \times 6=354$ pairs are valid. Similarly, we generate $236 \times 4,080=962,880$ and $236 \times 1,335=315,060$ invalid samples, that consist of Nexus images coupled with ALOI and Google images, respectively. Thus, each training fold has a total of 1, 305, 670 samples, of which 354 are valid.

Synthetic image attack datasets. We divide the synthetic image dataset of $\S 6.1$ into 2 equal sets, each containing 100,000 images. Then, we build two synthetic image attack datasets (DS1 and DS2) by repeating the following process for each subset of the synthetic image dataset: combine each Nexus dataset image, used as a reference image, with each image from the subset of the synthetic image dataset, used as a candidate image. Therefore, in total we have 140 million samples in each of DS1 and DS2.

Synthetic credential attack dataset. We use the synthetic credential dataset described in $\S 6.1$ to build a synthetic credential attack dataset: for each value of $\lambda$, combine the imageprint of each Nexus dataset image, used as a reference imageprint, with each imageprint in synthetic credential dataset, used as the candidate imageprint. Hence, we have 140 million authentication samples in this dataset for each value of $\lambda$. We repeat this process for 10 values of $\lambda$, ranging from 50 to 500 . Therefore, in total this dataset contains $10 \times 140 \mathrm{M}=1.4$ billion samples.

Illumination robustness evaluation dataset. To evaluate the performance of ai.lock under illumination changes, we use the ALOI holdout set $(3,600$ images) that includes up to 11 images of each object captured under a different illumination condition. Specifically, we pair each image in the ALOI holdout set (i.e., not used during training) with all the other images in this set. Therefore, we have a total of $\frac{3600 \times 3599}{2}=6,478,200$ authentication samples in the illumination robustness evaluation dataset, of which 6,306 samples are valid.

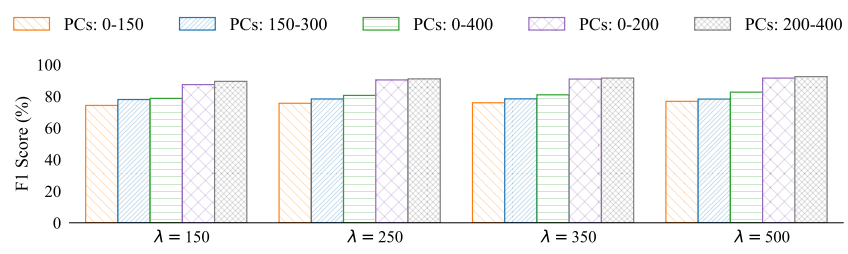

Figure 4: Comparison of ai.lock performance (F1 score) when using different subset of principal component feature ranks for different imageprint length $(\lambda)$ values. PCs ranked 200-400 constantly outperform other tested subsets.

Entropy evaluation dataset. We randomly selected 2 billion unique pairs of images from the YFCC100M toy dataset. In each pair, an image is considered to be the reference, the other is the candidate.

\section{EXPERIMENTAL EVALUATION}

We evaluate ai.lock and its variants. First, we describe the process we used to identify the best ai.lock parameters. We use these parameters to evaluate the performance of ai.lock under the attack datasets of $\S 6.2$. We also show that ai.lock is a $\delta$-LSIM function, empirically estimate its entropy, and measure its speed on a mobile device.

\section{1 ai.lock: Parameter Choice}

We identify the best parameters for the ai.lock variants using 5 fold cross validation on the ai.lock training dataset (see $\S 6.2$ ).

Best principal component range. To identify the best $\mathrm{PC}$ range, we use 5 fold cross validation as follows. First, we retrieve the embedding vector (output of the last hidden layer of Inception.v3) for each image in the ai.lock training dataset. Then, for each cross validation experiments, we use 4 training folds to find the principal components of the embedding vectors. Then, we transform the embedding vectors of the test fold into the newly identified feature space. Finally, we project them into several randomly generated vectors (LSH) to construct the binary imageprint of the images.

We have experimented with different subsets of the transformed feature space of various size including the first and second consecutive principal component sets of size 50,100,150, and 200, as well as, the first 400 PCs.

Figure 4 shows the cross validation performance achieved by ai.lock when using different subsets of PC features for different $\lambda$ values. We observe that the PCs ranked 200-400 perform consistently the best. This might seem surprising, as higher ranked PCs have higher variability and thus we expected that they would have more impact in differentiating between valid and invalid samples. We conjecture that some of the lower rank coordinates of these transformed vectors are more efficient in capturing the lower level details of the input object images that differentiate them from other object images.

Identify the best threshold. We identify the best threshold that separates the valid from the invalid authentication samples using the binary imageprints of the testing folds in each of the 5 cross validation experiments using ai.lock training set. Particularly, we normalize the Hamming distance of each pair of imageprints in the 


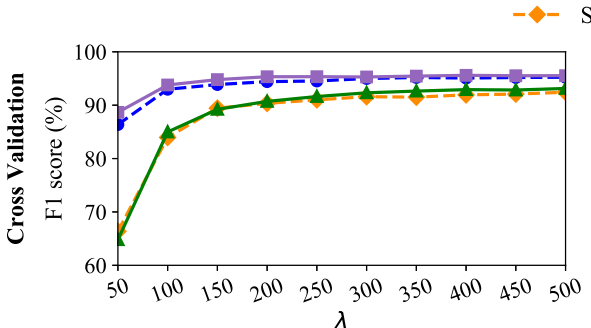

(a)

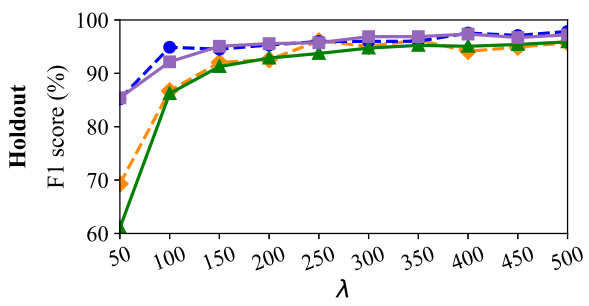

(d)

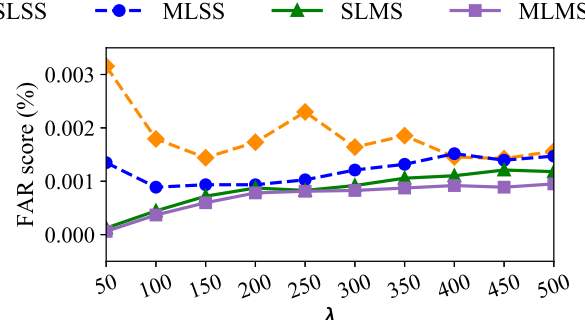

(b)

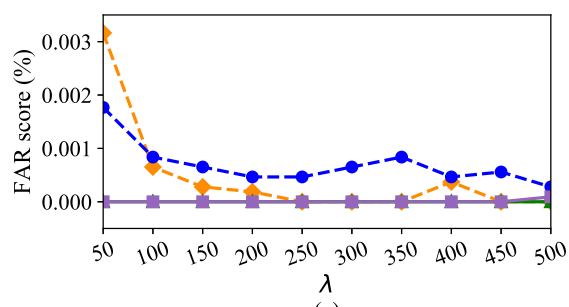

(e)

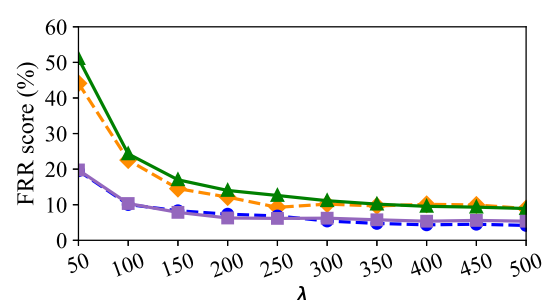

(c)

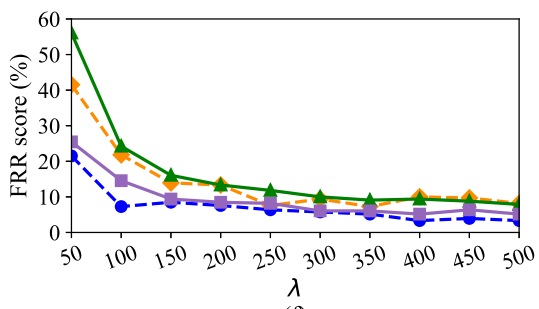

(f)

Figure 5: (a-c) ai.lock cross validation performance, and (d-f) ai.lock holdout performance using different ai.lock variants: Single Layer Single Segment (SLSS), Multi Layer Single Segment (MLSS), Single Layer Multi Segment (SLMS), Multi Layer Multi Segment (MLMS). Exploiting information from multiple Inception.v3 DNN layers (multi layer variants) lowers the FRR, while splitting images into smaller segments (multi segment variants) lowers the FAR. The MLMS variant of ai.lock consistently achieves the lowest FAR, that can be as low as $0 \%$ for the holdout dataset.

\begin{tabular}{c|cccccccccc}
\hline$\lambda$ & $\mathbf{5 0}$ & $\mathbf{1 0 0}$ & $\mathbf{1 5 0}$ & $\mathbf{2 0 0}$ & $\mathbf{2 5 0}$ & $\mathbf{3 0 0}$ & $\mathbf{3 5 0}$ & $\mathbf{4 0 0}$ & $\mathbf{4 5 0}$ & $\mathbf{5 0 0}$ \\
\hline$\tau \times 10$ & 7.80 & 7.30 & 7.07 & 6.95 & 6.80 & 6.87 & 6.80 & 6.85 & 6.87 & 6.82 \\
\hline
\end{tabular}

Table 3: Error tolerance threshold $(\tau)$ values for the basic ai.lock obtained through cross validation over the ai.lock dataset, when using PCs with feature ranked 200-400.

test fold by the length of the imageprints. Then, we apply more than $4 \mathrm{~K}$ different real values, between 0 and 1 , as a threshold on the normalized Hamming distances of the authentication pairs to classify them. At the end of the $5^{\text {th }}$ cross validation experiment, we select the threshold that has the maximum average performance, in terms of F1 score, as the best separating threshold. We call this the Error Tolerance Threshold, which we denote by $\tau$.

Table 3 reports the $\tau$ values for basic ai.lock with different values of $\lambda$, when using PCs with feature rank 200-400. We observe that as $\lambda$ increases, the value for $\tau$ decreases: we posit that larger $\lambda$ values preserve more information about the input vectors (PCs of the embedding vectors) in the LSH output.

We translate $\tau$ to the error correcting capacity required for ECC. Specifically, for an imageprint of length $\lambda$, we choose an ECC that is able to correct up to $c=\lfloor\lambda \times(1-\tau)\rfloor$ bits.

ai.lock MLSS variant. Similar to the basic ai.lock, we have experimented with multiple ranges of PCs and $\lambda$ values to identify the $\tau$ values for MLSS ai.lock, using the 5 fold cross validation experiment on the ai.lock training dataset.

ai.lock: Multi segment variants. For this ai.lock variant, we identify the $\tau$ values separately for each image segment, using the 5 cross validation experiment explained above. Therefore, we end up having 5 different $\tau$ values corresponding to each image segment. The $\tau$ corresponding to each segment can be used to identify if

\begin{tabular}{c|ccc}
\hline$t$ (matching segment counts out of 5) & $\mathbf{3}$ & 4 & 5 \\
\hline F1 score (\%) for SLMS & $\mathbf{9 3 . 1 3}$ & 90.95 & 85.84 \\
F1 score (\%) for MLMS & $\mathbf{9 5 . 5 3}$ & 94.64 & 92.42 \\
\hline
\end{tabular}

Table 4: Cross validation performance (F1 score) for different values of $t$ (number of segments that need to match out of 5) when using PCs with feature rank 200-400 and $\lambda=500$ for SLMS and MLMS variants of ai.lock. $t=3$ consistently achieves the best performance.

there is a match between the piece of the candidate image to the corresponding piece in the reference image. We say that the whole candidate and reference images match, when $t$ of their segments match. We have tested with $t$ ranging from 3 to 5 and observed that $t=3$ achieved the best F1 score (see Table 4).

Cross validation performance. We now report the cross validation performance of ai.lock with the parameters identified above, for $\lambda$ ranging from 50 to 500 . Figures 5 (a)-(c) compare the F1 score, FAR and FRR values of the best version of the ai.lock variants (basic SLSS, SLMS, MLSS, and MLMS) over the 5-fold cross validation experiments, using ai.lock training dataset. The performance of all ai.lock variants improves with increasing the value of $\lambda$. The MLMS ai.lock achieves the best performance, with an F1 score of $95.52 \%$ and FAR of $0.0009 \%$ when $\lambda=500$. The MLSS ai.lock also consistently improves over the basic ai.lock, with a smaller FRR and a smaller or at most equal FAR. Its FRR $(4.18 \%$ for $\lambda=500)$ is slightly smaller than that of MLMS variants (5.36\%), but it exhibits a slight increase in FAR. For large values of $\lambda$, the FRR of SLMS and SLSS are almost equivalent.

The average cross validation Equal Error Rate (EER, the rate at which the FAR = FRR) of ai.lock for the SLSS and MLSS variants is 


\begin{tabular}{c|ccccc}
\hline$\lambda$ & $\mathbf{5 0}$ & $\mathbf{1 5 0}$ & $\mathbf{2 5 0}$ & $\mathbf{3 5 0}$ & $\mathbf{5 0 0}$ \\
\hline $\mathrm{FAR} \times 10^{+6}$ & 33.87 & 4.34 & 3.29 & 0.69 & 0.20 \\
\hline
\end{tabular}

Table 5: SLSS ai.lock performance on synthetic attack DS1. The FAR decreases significantly as $\lambda$ grows from 50 to 500 . The FAR when $\lambda=\mathbf{5 0 0}$ is only $0.2 \times 10^{-6}$.

less than $0.67 \%$ and $0.17 \%$ respectively when using PCs with feature rank $200-400$ and $\lambda=500$.

The purpose of the LSH-based transformation is to encode the feature vector of an image extracted by a DNN into a binary string. Our conjecture is that larger lambda values extract more high quality information about the feature vectors, which in turn leads to lower FAR and FRR. This is partly due to the random nature of the LSH we used (see Figure 9), where roughly half of the bits among different images are different, and images of the same object have a smaller distance overall. Using more LSH bits reduces the variance of the distance that was due to perturbations from using a random projection, hence provides a better separation between TP and FP image comparisons.

\subsection{Resilience to Illumination Changes}

We evaluate the resilience of ai.lock to illumination changes using the $6,478,200$ authentication samples of the illumination robustness evaluation dataset ( $\$ 6.2)$. While the FAR of the MLMS variant of ai.lock (for $\lambda=500$ and $t=3$ ) remains very small $(0.006 \%$ ), its FRR increases to $16.9 \%$. Decreasing the required matching segments count $(t)$ to 2 , reduces the FRR to $11.43 \%$, which results in a slightly higher FAR of $0.010 \%$.

\section{3 ai.lock Under Attack}

Holdout dataset, real image attack. The performance over the ai.lock holdout set is reported in Figure 5(d)-(f). As before, the performance of all the ai.lock variants improves with the increase in $\lambda$. In agreement with the results of the cross validation experiments, we conclude that exploiting information from multiple Inception.v3 layers decreases the FRR, while using information from multiple image segments decreases the FAR. In addition, the MLMS ai.lock variant achieves the highest F1 score $(97.21 \%$ for $\lambda=500)$. The SLMS and MLMS schema consistently achieve the lowest FAR, which is as low as $0 \%$ on the holdout dataset.

Synthetic image attack. We use the synthetic attack dataset DS1 of $\S 6.2$ to evaluate the performance of SLSS ai.lock, using the trained parameters of $\S 7.1$. Table 5 shows the performance of ai.lock in classifying these attack samples. The FAR decreases significantly with $\lambda$, and is as low as $0.00002 \%$ when $\lambda=500$.

The proportion of the reference images that have been broken at least once decreases significantly by increasing $\lambda$ : from $16.86 \%$ to $0.79 \%$ (11 Nexus images) when $\lambda$ is 150 and 500 respectively. A majority of the broken references are broken only by a small number of candidate images: when $\lambda=500$, only 2 of the 11 broken images have been broken 5 times by the synthetic images in DS1. The average number of trials until finding the first matching synthetic image, over the 11 broken reference images, is 31,800 .

Vaccinated ai.lock. To further improve the ai.lock resistance to synthetic image attacks, we use the synthetic image attack dataset DS2 (see $\S 6.2$ ) along with the ai.lock training dataset, to train

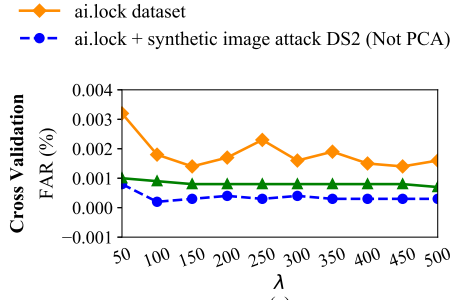

(a)

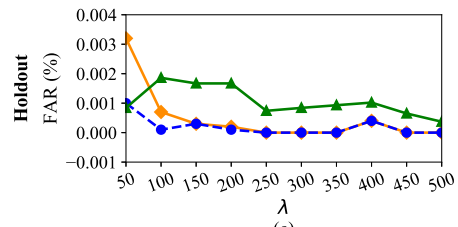

(c)
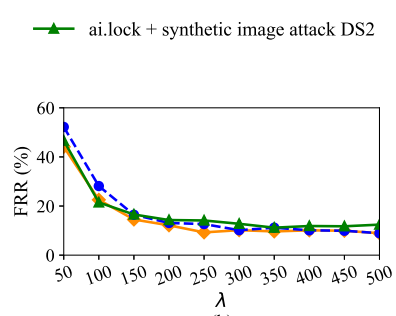

(b)

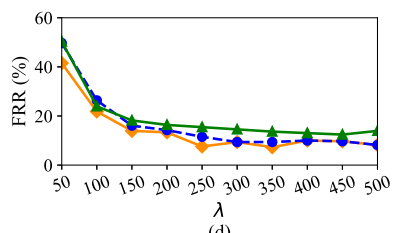

(d)
Figure 6: (a) Cross validation FAR, (b) Cross validation FRR , (c) Holdout FAR, and (d) Holdout FRR of SLSS ai.lock when trained over the ai.lock and synthetic image attacks of DS2.

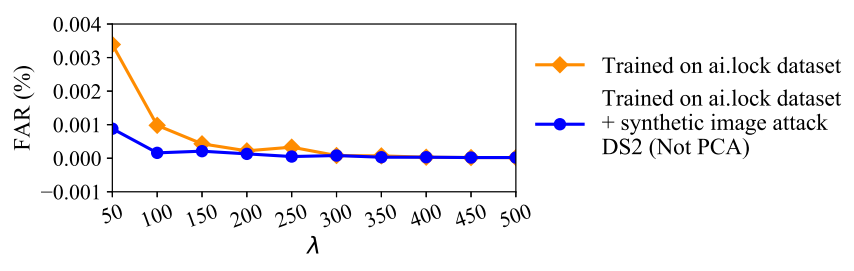

Figure 7: FAR of ai.lock on synthetic image attack, when trained on the ai.lock dataset vs. when trained also on DS2. The "vaccinated" ai.lock improves its resistance to the synthetic image attack: the FAR drops by more than $74 \%, 51 \%$ and $59 \%$ when $\lambda$ is 50,150 and 350 respectively.

ai.lock. Specifically, we divide the synthetic image attack dataset DS2 into 5 folds and distribute them into the 5 training folds of the ai.lock dataset. In other words, we train ai.lock on an additional $236 \times 20,000=4,720,000$ invalid authentication samples. The holdout set remains untouched and is used to evaluate the effectiveness of this approach. Then, we train ai.lock with SLSS as before using the cross validation experiment (see $\S 7.1$ ).

We experimented with two cases. First, the invalid synthetic image attack samples in DS2 contribute to both the PCA based feature selection and the error tolerant threshold $(\tau)$ discovery processes. Second, those samples are only used in the process of discovering $\tau$. Figure 6 shows the cross validation FAR and FRR (a, b) as well as the performance over the holdout set (c, d). In both experiments, we observed a drop in the FAR of ai.lock, however, the FRR increases. The FAR improvement is higher for the second case. We conjecture that the inclusion of synthetic, not camera captured images, is misleading the PCA based feature selection module into capturing irrelevant information.

We used the ai.lock trained on the synthetic image attack dataset DS2 to evaluate its performance over the synthetic image attack DS1. Figure 7 compares the performance of ai.lock when trained 


\begin{tabular}{c|ccccc}
\hline$\lambda$ & $\mathbf{5 0}$ & $\mathbf{1 5 0}$ & $\mathbf{2 5 0}$ & $\mathbf{3 5 0}$ & $\mathbf{5 0 0}$ \\
\hline $\mathrm{FAR} \times 10^{+6}$ & 11.89 & 0.09 & 0.03 & 0.000 & 0.000 \\
\hline
\end{tabular}

Table 6: SLSS ai.lock performance on the synthetic credential attack. ai.lock is unbreakable under 1.4 billion samples of the synthetic credential attack: its FAR is 0 when $\lambda \geq 300$.

\begin{tabular}{c|ccc}
\hline$\lambda$ & $\mathbf{1 5 0}$ & $\mathbf{3 5 0}$ & $\mathbf{5 0 0}$ \\
\hline$P_{1}$ & $8.6 \mathrm{e}-1$ & $9.3 \mathrm{e}-1$ & $9.1 \mathrm{e}-1$ \\
$P_{2}$ & $2.8 \mathrm{e}-6$ & 0.0 & 0.0 \\
\hline
\end{tabular}

Table 7: Average probability of collision, for valid $\left(P_{1}\right)$ and invalid $\left(P_{2}\right)$ samples in the ai.lock holdout set, when the ai.lock imageprint is considered as image hash value and at most $c=\lfloor\lambda \times(1-\tau)\rfloor$ bits of error is allowed. In all cases, $P_{1}>P_{2}$, thus conclude that ai.lock is an LSIM function.

on the ai.lock dataset and when trained on the ai.lock and the synthetic dataset DS2. Training also over synthetic image attack samples helps ai.lock to be more resilient to synthetic image attack, especially for small values of $\lambda$.

Synthetic credential attack. Table 6 shows the FAR values for ai.lock under the synthetic credential attack dataset described in $\S 6.2$. For all values of $\lambda$ greater than 300 , the FAR of ai.lock is equal to 0 . Even for a $\lambda$ of 50 , the FAR is $11.89 \times 10^{-4} \%$. This is an important result: even a powerful adversary who can create and test synthetic credentials on a large scale, is unable to break the ai.lock authentication.

\subsection{Is ai.lock $\delta$-LSIM?}

We now evaluate if the basic ai.lock (SLSS) variant, with the parameters identified in $\S 7.1$ preserves the similarity of the input space, i.e., if it satisfies the LSIM properties (see Definition 3.1). We use the ai.lock holdout set to evaluate the probability of obtaining the same hash value for valid and invalid samples.

Let $\pi_{i}$ and $\pi_{j}$ be the imageprints corresponding to two images in the ai.lock holdout set. Let $d_{H}\left(\pi_{i}, \pi_{j}\right)$ denote the Hamming distance and $S_{H}\left(\pi_{i}, \pi_{j}\right)$ denote the normalized Hamming similarity of these imageprints, i.e., $S_{H}\left(\pi_{i}, \pi_{j}\right)=1-\frac{d_{H}\left(\pi_{i}, \pi_{j}\right)}{\lambda}$.

The output of ai.lock can be considered either as a single bit or a string of bits. In the former case, the imageprints consist of the concatenation of the output of multiple hash functions, while in the later case, the entire imageprint is assumed to be the ai.lock hash value. In the following, we empirically evaluate the $P_{1}$ and $P_{2}$ values (see Definition §3.1), for the case where the entire ai.lock imageprint is considered as the hash value. In Appendix C, we further show that ai.lock is also a $\delta$-LSIM function when its hash value is a single bit.

We set $\delta=\tau$, where $\tau$ is the error tolerance threshold obtained from the ai.lock training process (see Table 3), for different values of $\lambda$. Table 7 shows the $P_{1}$ and $P_{2}$ values achieved by the basic ai.lock over the holdout dataset. We perform Mann-Whitney onesided test with alternative hypothesis $P 1>P 2$. Based on the observed $p-$ value $=0.00,(\alpha=0.05)$, for different values of $\lambda$, we conclude that the alternative hypothesis is true, hence, ai.lock is a $\delta$-LSIM function over the holdout dataset.

\begin{tabular}{c|cccc}
\hline$\lambda$ & $\mathbf{1 5 0}$ & $\mathbf{2 5 0}$ & $\mathbf{3 5 0}$ & $\mathbf{5 0 0}$ \\
\hline DI2E module (Inception v.1) & 0.7 & 0.7 & 0.7 & 0.7 \\
DI2E module (Inception v.3) & 1.9 & 1.9 & 1.9 & 1.9 \\
PCA + LSH module & 0.044 & 0.049 & 0.051 & 0.066 \\
\hline
\end{tabular}

Table 8: Processing time (in seconds) of SLSS ai.lock modules, for different values of $\lambda$. When using Inception.h5, the overall ai.lock speed is below $0.8 s$.

\subsection{On the Entropy of Imageprints}

We have used the entropy evaluation dataset (see $\S 6.2$ ) to empirically calculate the entropy of the imageprints generated by the ai.lock variants. The empirical entropy of an authentication solution is propositional to the size of the keyspace that the attacker needs to search to find a match for the authentication secret. For biometric information, estimating this size is difficult. In such cases, the entropy can be estimated as $-\log _{2}\left(\frac{1}{F A R}\right)$ [46]. We performed this study for different values of $\lambda$ and the best parameter choice of ai.lock (see $\S 7.1$ ), using the entropy evaluation dataset.

On the 2 billion image pairs in the entropy evaluation dataset, the FAR of the SLSS ai.lock variant is $0.020 \%$ and $0.035 \%$ when $\lambda$ is 50 and 500 respectively, for an entropy of 12.28 bits and 11.48 bits. We have visually inspected several hundreds of image pairs that resulted in false accepts and observed that a significant proportion were due to images that contained the same object type, e.g. ribbons, helmets, etc. This result is not unexpected: the SLSS variant uses only the last hidden layer of Inception.v3 network. Since Inception.v3 is trained for image classification task, it is expected to have similar activations on the last hidden layer for images of the same object type. We expect to eliminate this situation by requiring the match between activations of multiple inception layers (multi layer variant).

The FAR of the MLMS ai.lock variant on the entropy evaluation dataset, for $\lambda$ values of 500 and 150 , is $0.0007 \%$ and $0.0004 \%$ respectively. Therefore, the estimated entropy of ai.lock imageprints is 17.14 and 18.02 bits respectively.

\section{6 ai.lock Speed}

We have implemented ai.lock using Android 7.1.1 and Tensorflow 0.12 .1 and have evaluated its speed using 1,000 images of the Nexus dataset on a Nexus 6P smartphone (Qualcomm Snapdragon 810 CPU and 3GB RAM). Table 8 shows the average processing time of the 3 main ai.lock modules for different values of $\lambda$. Independent of the value for $\lambda$, ai.lock's DI2E module takes $1.9 \mathrm{~s}$ to compute the activations of all the layers of Inception.v3. When using Inception.h5 [63] (a smaller network), DI2E module takes 0.7s. The combined PCA and LSH speed increases with the value of $\lambda$, but is below $70 \mathrm{~ms}$ for $\lambda=500$. The processing overhead of ai.lock is below $2 \mathrm{~s}$ and $1 \mathrm{~s}$ using Inception.v3 and Inception.h5 respectively.

To minimize its impact on user experience on a Nexus 6P, ai.lock needs to use Inception.h5. The most significant processing overhead of ai.lock is on computing the activation of the DNN, which directly depends on the size of the network. Note that compressing the network using the DNN distillation approach [25] can alleviate this overhead. Future device and Inception improvements will likely improve the ai.lock performance and accuracy. 


\section{DISCUSSION AND LIMITATIONS}

Default authentication, revocation and recovery. If the image based authentication fails a number of times or the ai.lock secret is not available, the authentication falls back to the default authentication mechanism, e.g. text passwords.

Strong passwords. ai.lock benefits from users choosing strong, high-entropy and unique objects for authentication. ai.lock can use datasets of images of frequently occurring, thus low entropy, objects and learn to reject similar objects during their registration by the user. Further, the image classification task can be adapted to detect images belonging to classes of weak, low-entropy authentication objects. In addition, similar to text passwords, users could be encouraged to pick an ordered combination of personal objects for authentication.

Usability. Although usability is not the focus of this paper, we expect ai.lock to share several limitations with face based authentication mechanisms due to their similarities in the form factor. These include susceptibility to inappropriate lighting conditions [9]. While the FAR of ai.lock remains small under illumination changes, its FRR increases, affecting its usability. However, DNNs are capable of learning representations that are invariant to input changes, e.g. lighting, translation, etc. Thus, the DI2E module of ai.lock can be further fine-tuned to be more resistant to illumination changes. We leave the investigation of such improvement for future work.

In [6] we have evaluated the usability aspects of an image based authentication approach, and have shown that (1) the user entry time was significantly shorter compared to text passwords on a mobile device, (2) the participants were able to remember their authentication objects 2 and 7 days after registering them, and (3) the participants perceived object based authentication to be easier to use than text passwords, and were willing to adopt it. Further studies are required to understand (1) the user choice of the secret objects or scenes and whether it impacts the secret key space, (2) the ability of ai.lock to filter out common or low-entropy images, (3) the scenarios where users are willing to adopt ai.lock authentication and (4) other limitations associated to ai.lock authentication. Shoulder surfing. Similar to face based authentication, ai.lock is vulnerable to shoulder surfing attacks where the adversary captures images of the objects or scenes used by victims. However, ai.lock eliminates remote attacks, e.g., [51], moves the target away from sensitive body features, and enables users to trivially change their image-passwords. Similar to biometrics, ai.lock can also benefit from liveness verification techniques [55], that ensure that the adversary has physical access to the authentication object or scene, to prevent sophisticated image replay attacks. In addition, in Appendix $B$ we show that the knowledge of the authentication object type does not provide the adversary with significant advantage when launching a brute force attack.

Multi-factor authentication. ai.lock can also be used in conjunction with other authentication solutions. For instance, the image password set and authentication steps described in $\S 4.1$ can take advantage of a secondary secret (e.g. password, PIN), increasing the number of authentication factors to improve security. To this end, let $r$ be a random salt. We modify $x$ in the fuzzy biometric protection solution outlined in $\S 4.1$ to be the randomized hash of the secondary secret computed using salt $r$. Randomized hashing ensures the required formatting and bit length for $x$ can be achieved using key derivation function (e.g. HKDF [36]), etc. The random salt $r$ needs to be stored along with the other authentication credentials, i.e. $S S(R, x)$.

Compromised device. Our model assumes an adversary that physically captures a victimfis device and thus has black-box access to the authentication function. ai.lock is not resilient to an adversary who installs malware on the victim device. Such malware may for instance leverage PlaceRaider [66] to construct three dimensional models of the environment surrounding the victim, including of the authentication object.

Trusted hardware can secure ai.lock and even obviate the need for secure sketches. However, it would reduce the number of devices where ai.lock can be applied. Techniques similar to AuDroid [50] could be employed to ensure that unauthorized processes or external parties cannot access and misuse the device camera, however, they may still leave ai.lock vulnerable to cache attacks [38].

\section{CONCLUSIONS}

In this paper, we introduced ai.lock, the first secure and efficient image based authentication system. We have presented a suite of practical yet powerful image based attacks and built large scale attack datasets. We have shown that even under our powerful attacks, ai.lock achieves better entropy than state-of-the-art biometric authentication solutions.

\section{ACKNOWLEDGMENTS}

We thank the shepherd and reviewers for their excellent feedback. This research was supported in part by grants from the NSF (CNS1526494, CNS-1527153 and SES-1450619) and the Florida Center for Cybersecurity.

\section{REFERENCES}

[1] 2015. Replacing RSA SecurID: Why Are Customers Switching to Duo Security? (2015). https://duo.com/blog/replacing-rsa-securid-why-are-customersswitching-to-duo-security.

[2] 2017. TAPS - Make touchscreen gloves using a Sticker w/ Touch ID. (2017). https://www.kickstarter.com/projects/nanotips/taps-touchscreensticker-w-touch-id-ships-before-x?token=5b586aa6.

[3] Martín Abadi, Paul Barham, Jianmin Chen, Zhifeng Chen, Andy Davis, Jeffrey Dean, Matthieu Devin, Sanjay Ghemawat, Geoffrey Irving, Michael Isard, Manjunath Kudlur, Josh Levenberg, Rajat Monga, Sherry Moore, Derek G. Murray, Benoit Steiner, Paul Tucker, Vijay Vasudevan, Pete Warden, Martin Wicke, Yuan Yu, and Xiaoqiang Zheng. 2016. TensorFlow: A System for Large-Scale Machine Learning. In 12th USENIX Symposium on Operating Systems Design and Implementation (OSDI 16). USENIX Association, GA, 265-283. https://www.usenix.org/conference/osdi16/technical-sessions/presentation/abadi

[4] Apple Support 2017. About Touch ID security on iPhone and iPad. (2017). https://support.apple.com/en-us/HT204587.

[5] Sunpreet S Arora, Kai Cao, Anil K Jain, and Nicholas G Paulter. 2014. 3D Fingerprint Phantoms. In 2014 22nd International Conference on Pattern Recognition. 684-689. DOI : https://doi.org/10.1109/ICPR.2014.128

[6] Mozhgan Azimpourkivi, Umut Topkara, and Bogdan Carbunar. 2017. Camera Based Two Factor Authentication Through Mobile and Wearable Devices. In ACM International foint Conference on Pervasive and Ubiquitous Computing.

[7] Yu Bai, Li Zhuo, Bo Cheng, and Yuan Fan Peng. 2014. Surf feature extraction in encrypted domain. In 2014 IEEE International Conference on Multimedia and Expo (ICME). 1-6. DOI : https://doi.org/10.1109/ICME.2014.6890170

[8] Herbert Bay, Tinne Tuytelaars, and Luc Van Gool. 2006. SURF: Speeded Up Robust Features. Springer Berlin Heidelberg, Berlin, Heidelberg, 404-417. DOI : https://doi.org/10.1007/11744023_32

[9] Chandrasekhar Bhagavatula, Blase Ur, Kevin Iacovino, Su Mon Kywe, Lorrie Faith Cranor, and Marios Savvides. 2015. Biometric authentication on iphone 
and android: Usability, perceptions, and influences on adoption. Proceeding of Usable Security (USEC) (2015), 1-2.

[10] Joseph Bonneau. 2012. The Science of Guessing: Analyzing an Anonymized Corpus of 70 Million Passwords. In 2012 IEEE Symposium on Security and Privacy. 538-552. DOI : https://doi.org/10.1109/SP.2012.49

[11] Raj Chandra Bose and Dwijendra K Ray-Chaudhuri. 1960. On a class of error correcting binary group codes. Information and Control 3, 1 (1960), 68 - 79. DOI https://doi.org/10.1016/S0019-9958(60)90287-4

[12] Moses S. Charikar. 2002. Similarity Estimation Techniques from Rounding Algorithms. In Proceedings of the Thiry-fourth Annual ACM Symposium on Theory of Computing (STOC '02). ACM, New York, NY, USA, 380-388. DOI: https://doi.org/10.1145/509907.509965

[13] S. Chopra, R. Hadsell, and Y. LeCun. 2005. Learning a similarity metric discriminatively, with application to face verification. In 2005 IEEE Computer Society Conference on Computer Vision and Pattern Recognition (CVPR'05), Vol. 1. 539546 vol. 1. DOI : https://doi.org/10.1109/CVPR.2005.202

[14] Ed. D. Hardt. 2012. The OAuth 2.0 Authorization Framework. RFC 6749. IETF. http://tools.ietf.org/html/rfc6749

[15] Darren Davis, Fabian Monrose, and Michael K. Reiter. 2004. On User Choice in Graphical Password Schemes. In Proceedings of the 13th Conference on USENIX Security Symposium - Volume 13 (SSYM'04). USENIX Association, Berkeley, CA, USA, 11-11. http://dl.acm.org/citation.cfm?id=1251375.1251386

[16] Jia Deng, Wei Dong, Richard Socher, Li-Jia Li, Kai Li, and Li Fei-Fei 2009. Imagenet: A large-scale hierarchical image database. In IEEE Conference on Computer Vision and Pattern Recognition. IEEE, 248-255. DOI https://doi.org/10.1109/CVPR.2009.5206848

[17] Yevgeniy Dodis, Leonid Reyzin, and Adam Smith. 2004. Fuzzy Extractors: How to Generate Strong Keys from Biometrics and Other Noisy Data. Springer Berlin Heidelberg, Berlin, Heidelberg, 523-540. DOI https://doi.org/10.1007/978-3-540-24676-3_31

[18] Jeff Donahue, Yangqing Jia, Oriol Vinyals, Judy Hoffman, Ning Zhang, Eric Tzeng, and Trevor Darrell. 2014. DeCAF: A Deep Convolutional Activation Feature for Generic Visual Recognition.. In Icml, Vol. 32. 647-655.

[19] Nathaniel Wesley Filardo and Giuseppe Ateniese. 2012. High-Entropy Visual Identification for Touch Screen Devices. Springer Berlin Heidelberg, Berlin, Heidelberg, 182-198. DOI : https://doi.org/10.1007/978-3-642-29101-2_13

[20] Karl Pearson F.R.S. 1901. LIII. On lines and planes of closest fit to systems of points in space. Philosophical Magazine Series 62 , 11 (1901), 559-572. DOI: https://doi.org/10.1080/14786440109462720 arXiv:http://dx.doi.org/10.1080/14786440109462720

[21] Javier Galbally, Arun Ross, Marta Gomez-Barrero, Julian Fierrez, and Javier Ortega-Garcia. 2012. From the iriscode to the iris: A new vulnerability of iris recognition systems. Black Hat Briefings USA (2012).

[22] Jan-Mark Geusebroek, Gertjan J. Burghouts, and Arnold W.M. Smeulders. 2005. The Amsterdam Library of Object Images. International fournal of Computer Vision 61, 1 (2005), 103-112. DOI: https://doi.org/10.1023/B:VISI.0000042993.50813.60

[23] Ian Goodfellow, Jean Pouget-Abadie, Mehdi Mirza, Bing Xu, David Warde-Farley, Sherjil Ozair, Aaron Courville, and Yoshua Bengio. 2014. Generative Adversarial Nets. In Advances in Neural Information Processing Systems 27, Z. Ghahramani, M. Welling, C. Cortes, N. D. Lawrence, and K. Q. Weinberger (Eds.). Curran Associates, Inc., 2672-2680. http://papers.nips.cc/paper/5423-generative-adversarial-nets.pdf

[24] Eiji Hayashi, Bryan Pendleton, Fatih Ozenc, and Jason Hong. 2012. WebTicket: Account Management Using Printable Tokens. In Proceedings of the SIGCHI Conference on Human Factors in Computing Systems (CHI '12). ACM, New York, NY, USA, 997-1006. DOI: https://doi.org/10.1145/2207676.2208545

[25] Geoffrey Hinton, Oriol Vinyals, and Jeff Dean. 2015. Distilling the knowledge in a neural network. arXiv preprint arXiv:1503.02531 (2015)

[26] Alexis Hocquenghem. 1959. Codes correcteurs d'erreurs. Chiffres 2, 147-156 (1959), 8-5.

[27] Chao-Yung Hsu, Chun-Shien Lu, and Soo-Chang Pei. 2012. Image Feature Extraction in Encrypted Domain With Privacy-Preserving SIFT. IEEE Transactions on Image Processing 21, 11 (Nov 2012), 4593-4607. DOI: https://doi.org/10.1109/TIP.2012.2204272

[28] Piotr Indyk and Rajeev Motwani. 1998. Approximate Nearest Neighbors: Towards Removing the Curse of Dimensionality. In Proceedings of the Thirtieth Annual ACM Symposium on Theory of Computing (STOC '98). ACM, New York, NY, USA, 604-613. DOI : https://doi.org/10.1145/276698.276876

[29] Anil K. Jain, Karthik Nandakumar, and Abhishek Nagar. 2008. Biometric Template Security. EURASIP 7. Adv. Signal Process 2008, Article 113 (Jan. 2008), 17 pages. DOI: https://doi.org/10.1155/2008/579416

[30] Jeff Kent. 2017. python-bchlib. (2017). https://github.com/jkent/python-bchlib/

[31] A. Juels and M. Sudan. 2002. A fuzzy vault scheme. In Proceedings IEEE International Symposium on Information Theory, 408-. DOI: https://doi.org/10.1109/ISIT.2002.1023680
[32] Ari Juels and Martin Wattenberg. 1999. A Fuzzy Commitment Scheme. In Proceedings of the 6th ACM Conference on Computer and Communications Security (CCS '99). ACM, New York, NY, USA, 28-36. DOI: https://doi.org/10.1145/319709.319714

[33] Nikolaos Karapanos, Claudio Marforio, Claudio Soriente, and Srdjan Čapkun. 2015. Sound-proof: Usable Two-factor Authentication Based on Ambient Sound. In Proceedings of the 24th USENIX Conference on Security Symposium (SEC'15). USENIX Association, Berkeley, CA, USA, 483-498. http://dl.acm.org/citation.cfm?id=2831143.2831174

[34] Thomas P Keenan. 2015. Hidden Risks of Biometric Identifiers and How to Avoid Them. BlackHat USA (2015).

[35] Klaus Kollreider, Hartwig Fronthaler, and Josef Bigun. 2008. Verifying liveness by multiple experts in face biometrics. In 2008 IEEE Computer Society Conference on Computer Vision and Pattern Recognition Workshops. 1-6. DOI : https://doi.org/10.1109/CVPRW.2008.4563115

[36] H. Krawczyk and P. Eronen. 2010. HMAC-based Extract-and-Expand Key Derivation Function (HKDF). RFC 5869. IETF. https://tools.ietf.org/html/rfc5869

[37] N. Lalithamani and M. Sabrigiriraj. 2015. Palm and hand vein-based fuzzy vault generation scheme for multibiometric cryptosystem. The Imaging Science fournal 63, 2 (2015), 111-118. DOI : https://doi.org/10.1179/1743131x14Y.0000000090 arXiv:http://dx.doi.org/10.1179/1743131x14Y.0000000090

[38] Moritz Lipp, Daniel Gruss, Raphael Spreitzer, Clémentine Maurice, and Stefan Mangard. 2016. ARMageddon: Cache Attacks on Mobile Devices. In 25th USENIX Security Symposium. 549-564.

[39] David G. Lowe. 2004. Distinctive Image Features from Scale-Invariant Keypoints. International fournal of Computer Vision 60, 2 (2004), 91-110. DOI: https://doi.org/10.1023/B:VISI.0000029664.99615.94

[40] Emanuele Maiorana, Patrizio Campisi, Julian Fierrez, Javier Ortega-Garcia, and Alessandro Neri. 2010. Cancelable Templates for Sequence-Based Biometrics with Application to On-line Signature Recognition. IEEE Transactions on Systems, Man, and Cybernetics - Part A: Systems and Humans 40, 3 (May 2010), 525538. DOI : https://doi.org/10.1109/TSMCA.2010.2041653

[41] J. M. McCune, A. Perrig, and M. K. Reiter. 2005. Seeing-is-believing: using camera phones for human-verifiable authentication. In 2005 IEEE Symposium on Security and Privacy (S P'05). 110-124. DOI : https://doi.org/10.1109/SP.2005.19

[42] D. Menotti, G. Chiachia, A. Pinto, W. R. Schwartz, H. Pedrini, A. X. Falco, and A. Rocha. 2015. Deep Representations for Iris, Face, and Fingerprint Spoofing Detection. IEEE Transactions on Information Forensics and Security 10, 4 (April 2015), 864-879. DOI : https://doi.org/10.1109/TIFS.2015.2398817

[43] Sanjeev Miglani and Manoj Kumar. 2016. India's billion-member biometric database raises privacy fears. (March 2016). http://www.reuters.com/article/usindia-biometrics-idUSKCNOWI14E.

[44] Karthik Nandakumar, Abhishek Nagar, and Anil K. Jain. 2007. Hardening Fingerprint Fuzzy Vault Using Password. Springer Berlin Heidelberg, Berlin, Heidelberg, 927-937. DOI : https://doi.org/10.1007/978-3-540-74549-5_97

[45] Douglas L Nelson, Valerie S Reed, and John R Walling. 1976. Pictorial superiority effect. Fournal of Experimental Psychology: Human Learning and Memory 2, 5 (1976), 523.

[46] Lawrence O'Gorman. 2003. Comparing passwords, tokens, and biometrics for user authentication. Proc. IEEE 91, 12 (Dec 2003), 2021-2040. DOI: https://doi.org/10.1109/JPROC.2003.819611

[47] Thian Song Ong, Andrew Teoh Beng Jin, and David Chek Ling Ngo. 2008. Application-Specific Key Release Scheme from Biometrics. If Network Security 6, 2 (2008), 127-133.

[48] K. Patel, H. Han, and A. K. Jain. 2016. Secure Face Unlock: Spoof Detection on Smartphones. IEEE Transactions on Information Forensics and Security 11, 10 (Oct 2016), 2268-2283. DOI : https://doi.org/10.1109/TIFS.2016.2578288

[49] Andrea Peterson. 2015. OPM says 5.6 million fingerprints stolen in cyberattack, five times as many as previously thought. (September 2015). https:/www.washingtonpost.com/news/the-switch/wp/2015/09/23/opm-nowsays-more-than-five-million-fingerprints-compromised-in-breaches/.

[50] Giuseppe Petracca, Yuqiong Sun, Trent Jaeger, and Ahmad Atamli. 2015. AuDroid: Preventing Attacks on Audio Channels in Mobile Devices. In Proceedings of the 31st Annual Computer Security Applications Conference. 181-190.

[51] Iasonas Polakis, Marco Lancini, Georgios Kontaxis, Federico Maggi, Sotiris Ioannidis, Angelos D. Keromytis, and Stefano Zanero. 2012. All Your Face Are Belong to Us: Breaking Facebook's Social Authentication. In Proceedings of the 28th Annual Computer Security Applications Conference (ACSAC '12). ACM, New York, NY, USA, 399-408. DOI : https://doi.org/10.1145/2420950.2421008

[52] S. Pouyanfar, S. C. Chen, and M. L. Shyu. 2017. An efficient deep residual-inception network for multimedia classification. In 2017 IEEE International Conference on Multimedia and Expo (ICME). 373-378. DOI: https://doi.org/10.1109/ICME.2017.8019447

[53] Zhan Qin, Jingbo Yan, Kui Ren, Chang Wen Chen, and Cong Wang. 2014. Towards Efficient Privacy-preserving Image Feature Extraction in Cloud Computing. In Proceedings of the 22Nd ACM International Conference on Multimedia (MM '14). ACM, New York, NY, USA, 497-506. DOI: https://doi.org/10.1145/2647868.2654941 
[54] Alec Radford, Luke Metz, and Soumith Chintala. 2015. Unsupervised representation learning with deep convolutional generative adversarial networks. arXiv preprint arXiv:1511.06434 (2015).

[55] M. Rahman, M. Azimpourkivi, U. Topkara, and B. Carbunar. 2017. Video Liveness for Citizen Journalism: Attacks and Defenses. IEEE Transactions on Mobile Computing PP, 99 (2017), 1-1. DOI : https://doi.org/10.1109/TMC.2017.2687922

[56] Nalini K. Ratha, Sharat Chikkerur, Jonathan H. Connell, and Ruud M Bolle. 2007. Generating Cancelable Fingerprint Templates. IEEE Trans. Pattern Anal. Mach. Intell. 29, 4 (April 2007), 561-572. DOI : https://doi.org/10.1109/TPAMI.2007.1004

[57] RSA SecurID 2017. RSA SecurID Suite. (2017). https://www.rsa.com/enus/products-services/identity-access-management/securid.

[58] Schlage Keypad Locks 2017. Schlage Keypad Locks User Guide. (2017) http://www.schlage.com/content/dam/sch-us/documents/pdf/installationmanuals/23780042.pdf.

[59] SecuriCode 2017. Ford SecuriCode Keyless Entry Keypad. (2017). http://owner.ford.com/how-tos/vehicle-features/locks-andsecurity/securicode-keyless-entry-keypad.html.

[60] Claude Elwood Shannon. 2001. A mathematical theory of communication. ACM SIGMOBILE Mobile Computing and Communications Review 5, 1 (2001), 3-55.

[61] Ling Shao, Fan Zhu, and Xuelong Li. 2015. Transfer Learning for Visual Categorization: A Survey. IEEE Transactions on Neural Networks and Learning Systems 26, 5 (May 2015), 1019-1034. DOI : https://doi.org/10.1109/TNNLS.2014.2330900

[62] Ivo Sluganovic, Marc Roeschlin, Kasper B. Rasmussen, and Ivan Martinovic 2016. Using Reflexive Eye Movements for Fast Challenge-Response Authentication. In Proceedings of the 2016 ACM SIGSAC Conference on Computer and Communications Security (CCS '16). ACM, New York, NY, USA, 1056-1067. DOI : https://doi.org/10.1145/2976749.2978311

[63] Christian Szegedy, Wei Liu, Yangqing Jia, Pierre Sermanet, Scott Reed, Dragomir Anguelov, Dumitru Erhan, Vincent Vanhoucke, and Andrew Rabinovich. 2015 Going Deeper With Convolutions. In The IEEE Conference on Computer Vision and Pattern Recognition (CVPR).

[64] C. Szegedy, V. Vanhoucke, S. Ioffe, J. Shlens, and Z. Wojna. 2016. Rethinking the Inception Architecture for Computer Vision. In 2016 IEEE Conference on Computer Vision and Pattern Recognition (CVPR). 2818-2826. DOI: https://doi.org/10.1109/CVPR.2016.308

[65] Y. Taigman, M. Yang, M. Ranzato, and L. Wolf. 2014 . DeepFace: Closing the Gap to Human-Level Performance in Face Verification. In 2014 IEEE Conference on Computer Vision and Pattern Recognition. 1701-1708. DOI: https://doi.org/10.1109/CVPR.2014.220

[66] Robert Templeman, Zahid Rahman, David J. Crandall, and Apu Kapadia. 2013. PlaceRaider: Virtual Theft in Physical Spaces with Smartphones. In Annual Network and Distributed System Security Symposium.

[67] tensorflow. 2017. Inception in TensorFlow. (2017). Retrieved May 5, 2017 from https://github.com/tensorflow/models/tree/master/inception

[68] Andrew B. J. Teoh, Alwyn Goh, and David C. L. Ngo. 2006. Random Multispace Quantization As an Analytic Mechanism for BioHashing of Biometric and Random Identity Inputs. IEEE Transactions on Pattern Analysis and Machine Intelligence 28, 12 (Dec. 2006), 1892-1901. DOI: https://doi.org/10.1109/TPAMI.2006.250

[69] Bart Thomee, David A. Shamma, Gerald Friedland, Benjamin Elizalde, Kar $\mathrm{Ni}$, Douglas Poland, Damian Borth, and Li-Jia Li. 2016. YFCC100M: The New Data in Multimedia Research. Commun. ACM 59, 2 (Jan. 2016), 64-73. DOI: https://doi.org/10.1145/2812802

[70] O. Wang, S. Hu, J. Wang, and K. Ren. 2016. Secure Surfing: PrivacyPreserving Speeded-Up Robust Feature Extractor. In 2016 IEEE 36th International Conference on Distributed Computing Systems (ICDCS). 700-710. DOI : https://doi.org/10.1109/ICDCS.2016.84

[71] Yi Xu, True Price, Jan-Michael Frahm, and Fabian Monrose. 2016. Virtual U: Defeating Face Liveness Detection by Building Virtual Models from Your Public Photos. In 25th USENIX Security Symposium (USENIX Security 16). USENIX Association, Austin, TX, 497-512.

[72] Jason Yosinski, Jeff Clune, Yoshua Bengio, and Hod Lipson. 2014. How Transferable Are Features in Deep Neural Networks?. In Proceedings of the 27th International Conference on Neural Information Processing Systems (NIPS'14). MIT Press, Cambridge, MA, USA, 3320-3328. http://dl.acm.org/citation.cfm?id=2969033.2969197

[73] Matthew D. Zeiler and Rob Fergus. 2014. Visualizing and Understanding Convolutional Networks. Springer International Publishing, Cham, 818-833. DOI https://doi.org/10.1007/978-3-319-10590-1_53

\begin{tabular}{c|cccc}
\hline \# of words in image search query & $\mathbf{1}$ & $\mathbf{2}$ & $\mathbf{3}$ & $\mathbf{4}$ \\
\hline Dataset size $\left(\boldsymbol{d}_{\text {size }}\right)$ & 12,413 & 24,882 & 26,418 & 26,766 \\
Avg \# of trials before FA (random order) & 12,078 & 23,205 & 24,641 & 25,028 \\
Avg \# of trials before FA (guessing attack) & 12,034 & 22,755 & 23,921 & 24,488 \\
\hline Portion of broken references (\%) & 5.0 & 9.0 & 10.9 & 9.0 \\
\hline
\end{tabular}

Table 9: ai.lock under the object guessing attack. The average number of trials before the first false accept (FA) drops only slightly in the object guessing attack scenario when compared to a random ordering of attack images. Thus, knowledge of the authentication object type provides the adversary only nominal guessing advantage.

\section{A MOTIVATION FOR FEATURE SELECTION USING PCA}
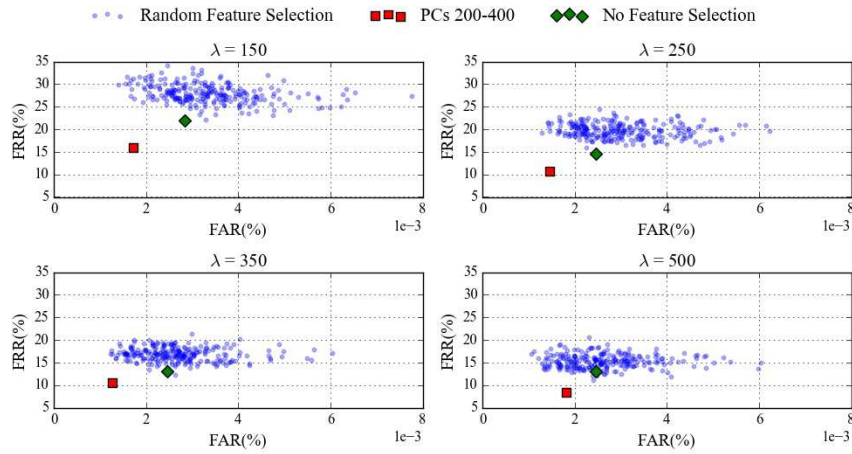

Figure 8: PCA motivation: FRR vs. FAR of (i) ai.lock when using PCA (with features ranked 200-400), (ii) ai.lock with no feature selection ("Raw"), and (iii) 250 independent instances of ai.lock when using a feature selection approach that randomly selects 200 features. ai.lock with PCA consistently achieves the lowest FRR and often the lowest FAR.

We now justify the need for the PCA step of ai.lock. For this, we compare the best version of ai.lock running PCA (i.e., features ranked 200-400), with two other versions. First, we consider a baseline version (which we call "Raw"), that uses no feature selection component. Specifically, Raw applies LSH to the raw embedding vectors, then, identifies the best threshold $\tau$ using the 5-fold cross validation experiment described in $\S 7.1$ for ai.lock. Second, we compare against an ai.lock variant where we replace the PCA component with a random choice of 200 features (of the embedding vectors) produced by the last hidden layer of Inception.v3. Figure 8 shows the results of this comparison for $\lambda$ values of 150, 250, 350 and 500, and 250 different instances of ai.lock with random feature selection. We observe that ai.lock with PCs of rank 200-400 consistently achieves the significantly lower FRR, and often the lowest FAR. In addition, we observe that randomly choosing the features is not ideal, as it often performs worse than when no feature selection is used at all.

\section{B OBJECT/SCENE GUESSING ATTACK}

Data. We have asked a graduate student to tag each of the 55 unique object images in the Nexus holdout set with 1 to 4 words. For each value of the number of tags per image (i.e., 1 to 4 ), and 


\begin{tabular}{c|ccc}
\hline$\lambda$ & $\mathbf{1 5 0}$ & $\mathbf{3 5 0}$ & $\mathbf{5 0 0}$ \\
\hline$P_{1}$ & 0.799 & 0.797 & 0.796 \\
$P_{2}$ & 0.500 & 0.500 & 0.500 \\
\hline
\end{tabular}

Table 10: Average probability of collision, for valid $\left(P_{1}\right)$ and invalid $\left(P_{2}\right)$ samples in the ai.lock holdout set per imageprint bit basis. In all cases, $P_{1}>P_{2}$, thus conclude that ai.lock with single bit hash value is an LSIM function.

each object image, we collected 300-500 images provided by Google's image search engine. Thus, we generated 4 Google image datasets, one for images found when searching with 1 tag, another when searching with 2 tags, etc. In total, we have collected 90,479 images.

ai.lock performance under object guessing attack. We use the 4 collected image datasets from Google to generate a total of 19, 905, 380 "guessing attack" authentication samples, and use them to evaluate the guessing entropy [15] of ai.lock under an object/scene guessing attack (see $\S 2$ ).

Specifically, using each of the 4 Google image datasets we perform the following two brute force attacks. The first attack emulates an object guessing attack: re-order the images in the Google dataset to start the brute force attack with the images of the same object type, then continue with images of other object categories in a random order. Finally, count the number of trials before the first match (false accept) occurs. The second attack is a standard brute force attack: randomly shuffle the images in the Google image dataset and use them to brute force each image in the Nexus holdout set. We use the second attack as a baseline, to determine if knowledge of the object type impacts the trial count to success. In both attacks, we count each of the unbreakable reference images as "success" at $d_{\text {size }}$ trials, where $d_{\text {size }}$ is the number of images in the corresponding Google image dataset (see Table 9).

Table 9 summarizes the ai.lock performance under the object/scene guessing attack scenario. We observe an increase in the portion of the Nexus images that are broken when the simulated adversary uses more words to describe the authentication objects for collecting the attack image dataset. However, for all experiments, the average number of trials before success drops only slightly in the object guessing attack scenario compared to the baseline. This is due to the fact that the reference images were mostly broken with images of different object categories. We conclude that knowledge of the secret object type does not provide the adversary with a significant guessing advantage.

\section{IS AI.LOCK $\delta$-LSIM FOR AI.LOCK WITH SINGLE BIT HASH VALUE?}

We now show that ai.lock with a single bit hash value is a $\delta$-LSIM (see Definition 3.1).

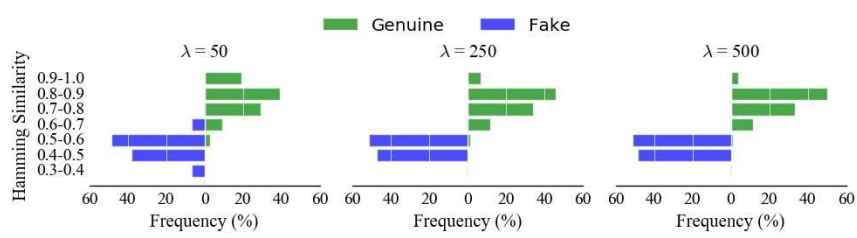

Figure 9: Histograms of normalized Hamming similarity between imageprints of valid and invalid authentication samples in the ai.lock holdout set. The red rectangles pinpoint the focus areas: valid samples with Hamming similarity below 0.6 and invalid samples with Hamming similarity above 0.6. Higher values of $\lambda$ provide more effective separation between valid and invalid samples: when $\lambda=500$, no invalid samples have similarity above 0.6 .

ai.lock uses Charikar's random projection LSH [12]. Therefore, for any embedding vector (the input to LSH function) $u$ and $v$, $\operatorname{Pr}[1$ bit collision $]=1-\frac{\theta(u, v)}{\pi}$, where $\theta(u, v)$ denotes the angle between $u$ and $v$. We use the angle between the feature vectors of images in the ai.lock holdout set to compute the average probability of collision: 0.79 for valid samples and 0.50 for invalid authentication samples.

Figure 9 shows the histogram of normalized Hamming similarity between imageprints in the valid and invalid samples of the ai.lock holdout set. Unsurprisingly, most invalid samples have a Hamming similarity between 0.4 and 0.6: different images have imageprints that are similar in around half of their bits (see also Table 10). We observe that the overlap between the Hamming similarities of valid and invalid samples significantly reduces for higher values of $\lambda$.

In addition, we compute these probabilities empirically by counting the number of times when the hash values collide for valid and invalid samples, after the LSH transformation. We then use this count to compute the average probability of collision for a valid $\left(P_{1}\right)$ and invalid $\left(P_{2}\right)$ authentication samples (see Table 10). We observe the remarkable similarity of these values, to the ones above, computed analytically. As $\lambda$ increases, the empirical $P_{1}$ approaches the analytic lower bound (0.79). We perform a MannWhitney one-sided test with alternative hypothesis $P 1>P 2$. This test suggests that there is a significant gap between $P_{1}$ and $P_{2}$ $(p-$ value $=0.00, \alpha=0.05)$ for all cases, hence, ai.lock is a $\delta$-LSIM on the Nexus holdout dataset.

In addition, comparing the values for $P 1$ and $P 2$ with the ones reported in $\S 7.4$ for ai.lock with multi-bit hash value, we observe that concatenating multiple hashes enlarges the gap between $P_{1}$ and $P_{2}$ values. 